\title{
The mating system of the fungus Cryphonectria parasitica: selfing and self-incompatibility
}

\author{
ROBERT E. MARRA* \& MICHAEL G. MILGROOM \\ Department of Plant Pathology, 334 Plant Sciences Building, Cornell University, Ithaca NY 14853, U.S.A.
}

\begin{abstract}
Although the genetic components of mating systems in fungi are well understood as laboratory phenomena, surprisingly little is known about their function in nature or about their role in determining mating patterns and population genetic structure. Our study of the mating system of the haploid ascomycete fungus, Cryphonectria parasitica, resulted in the following. (1) Laboratory crosses among 20 isolates, chosen randomly from North America and China, resolved into two incompatibility groups (occurring on both continents), confirming that $C$. parasitica has a diallelic, bipolar sexual self-incompatibility system, typical of other self-incompatible Ascomycetes, in which mating is only successful between isolates of opposite mating type. (2) PCR-based markers for mating-type alleles correlated perfectly with mating-type phenotypes of individual isolates. (3) Three genotypes, isolated from natural populations in Virginia and West Virginia, were inoculated onto chestnut trees in two sites in West Virginia and were confirmed to have self-fertilized and outcrossed in both sites. (4) Ten isolates, of a total of over 200 assayed, were confirmed to have self-fertilized in the laboratory, albeit at very low frequency. Five of these 10 isolates were ramets of a single genet, suggesting a genetic basis underlying the proclivity to self-fertilize in the laboratory. (5) Selffertilization could not be induced in the laboratory with exudates (ostensibly containing pheromones) from isolates of opposite mating type. These results demonstrate that, a sexual self-incompatibility system notwithstanding, self-fertilization occurs under both laboratory and field conditions in C. parasitica. The disparity between observations of frequent selfing in nature and rare selfing in the laboratory suggests that the mating system is under ecological as well as genetic control.
\end{abstract}

Keywords: chestnut blight, Cryphonectria parasitica, fungus, mixed mating, self-fertilization, selfincompatibility.

\section{Introduction}

Because of the near ubiquity of hermaphroditism in plants and fungi, the phenomena of self-compatibility and self-incompatibility are central to studies of plant and fungal mating systems. However, in contrast to more than a century of empirical and theoretical work addressing plant mating systems (reviewed in Barrett, 1988; Barrett \& Eckert, 1990; Jarne \& Charlesworth, 1993; Richards, 1997), the majority of fungal mating systems has yet to be investigated. Although a great deal of laboratory work has been focused on the mating systems of a few fungi (e.g. Saccharomyces cerevisiae, Neurospora crassa, Podospora anserina, and Coprinus

*Correspondence and present address: Department of Microbiology, Jones Building Box 3020, Duke University Medical Center, Durham NC 27710, U.S.A., Tel: 919684 9096, E-mail: r.marra@duke.edu cinereus), spatial and temporal dynamics of fungal mating systems in nature remain relatively unstudied. In those species where genetically determined selfincompatibility has been identified, it ranges from simple one-locus, two-allele systems to complex twolocus, multiallelic systems (Alexopoulos et al., 1996; p. 501). In the Ascomycota, sexual self-incompatibility is under the control of a single diallelic mating-type locus $(M A T)$, which controls the fertilization process, and subsequent steps in mating, as a regulator of diffusable and $M A T$-specific pheromones and receptors (Kues \& Casselton, 1992). Ascomycete self-incompatibility is considered 'bipolar'; that is, mating only occurs between individuals that have opposite alleles, or idiomorphs (MAT-1 and MAT-2) at this locus (Coppin et al., 1997; Kronstad \& Staben, 1997).

With the exception of a few studies of ascomycete and basidiomycete species (Raper, 1966; Rayner \& Turton, 
1982; Ainsworth, 1987; Ennos \& Swales, 1987; Leuchtmann \& Clay, 1989; Sharland \& Rayner, 1989a; Sharland \& Rayner, 1989b; Griffin et al., 1992; Kohli \& Kohn, 1992), little is known about the manner or degree to which the mating systems of fungi, as characterized in the laboratory, determine genetic structure in natural populations. The importance of studying fungal mating systems in both nature and the laboratory is demonstrated well by research on the ascomycete Cryphonectria parasitica, a fungus pathogenic on chestnut trees (Castanea dentata) in eastern North American forests. Mixed mating has been found in five populations in eastern North America, with outcrossing rates $(t)$ ranging from 0.68 to 0.73 (Marra, 1998; Milgroom et al., 1993).

The mating system of $C$. parasitica has so far eluded simple classification: both selfing and self-incompatibility have been claimed, but neither has been sufficiently documented. Significant amounts of self-fertilization occur in nature (see above), yet $C$. parasitica does not appear to self-fertilize readily in the laboratory (Marra, 1998; this study). A previous report of selfing in the laboratory (Puhalla \& Anagnostakis, 1971) cannot be considered conclusive, because controls for fertilization by contaminating male gametes (conidia) were not used, an essential control for demonstrating selfing unambiguously (Whitehouse, 1949). In marked contrast to the evidence for self-fertility in the field, it has been suggested (Anagnostakis, 1988) that C. parasitica is sexually self-incompatible, and recent work suggests that $C$. parasitica may have a self-incompatibility locus (MAT) similar to those of other self-incompatible ascomycetes (Marra \& Milgroom, 1999).

One explanation for this apparent contradiction is that what appears to be selfing in nature is not actually selfing, but biparental inbreeding to a degree that is beyond the resolution of available markers. In this case, absence of marker segregation may not be diagnostic of self-fertilization, but rather due to 'effective' or 'apparent' selfing (Hedrick, 1990). Spatial aggregation of genotypes (Milgroom \& Lipari, 1995b), and biparental inbreeding (Marra \& Milgroom, 1994; Marra, 1998) suggest that some populations may actually comprise relatively restricted genetic neighbourhoods (Wright, 1946). These alternative explanations for lack of marker segregation require that the hypothesis that self-fertilization occurs in nature be tested rigorously.

The possibility that a self-incompatible fungus is capable of self-fertilization warrants further examination, and is the motivation for our interest in establishing the genetics underlying self-incompatibility in the mating system in $C$. parasitica. However, because neither self-fertilization nor self-incompatibility has been sufficiently documented, our goals in this paper were (1) to demonstrate and describe the selfincompatibility system in $C$. parasitica, and (2) to determine if self-fertilization can in fact occur in the field and laboratory.

\section{Study system}

Cryphonectria parasitica, the ascomycete fungus that causes chestnut blight, was introduced into North America from Japan around 1900 (Milgroom et al., 1996), and spread rapidly across the natural range of the American chestnut (Castanea dentata), which currently persists as sprouts that regenerate from surviving root systems (reviewed in Anagnostakis, 1987).

The life cycle of the typical filamentous ascomycete is reviewed in Nelson (1996). C. parasitica infects chestnut trees and forms cankers that produce two types of spores: asexual (conidia) and sexual (ascospores). Conidia may function either as vegetative propagules or as male gametes in sexual reproduction. Fertilization in $C$. parasitica (as well as many other ascomycetes) occurs when a conidium and a receptive hypha (the female reproductive structure) fuse to form a dikaryon $(n+n)$. Synchronous nuclear divisions result in a cluster of dikaryotic hyphae within the developing fruit-body. Each terminal $n+n$ cell (ascus initial) within the fruit-body undergoes karyogamy, resulting in the brief diploid $(2 n)$ phase. Therefore, a single fertilization results in a fruitbody containing many $2 n$ cells. Each $2 n$ nucleus undergoes meiosis, followed by a single mitosis, resulting in eight ascospores per mature ascus. In $C$. parasitica, and all other members of the Class Pyrenomycetes, asci are borne inside a flaskshaped fruit-body, the perithecium. Dispersal occurs when ascospores (there may be thousands per perithecium) are forcibly ejected through an opening in the neck of the perithecium, which protrudes from the surface of the canker. Each haploid ascospore is dispersed by air currents, and is capable of infecting a tree and developing into a new canker, completing the fungus's life cycle.

\section{Materials and methods}

\section{Strains and isolates used}

We used a combination of field and laboratory-derived isolates of $C$. parasitica in this study. Unless otherwise specified, field isolates were collected from Mt. Lake, Virginia (Milgroom et al., 1993); Danby, New York; Depot Hill, New York (Milgroom \& Lipari, 1995a); and Parsons, West Virginia (Marra, 1998). Field isolates were obtained from bark samples (approx. $4 \mathrm{~cm}^{2}$ ) of chestnut trees with visible blight cankers. New laboratory 
strains originated as ascospores obtained from laboratory crosses. Culturing and storage of isolates were done according to standard protocols, as described in Cortesi et al. (1996) and Milgroom et al. (1993, 1992a,b).

\section{Crosses}

Crosses in the laboratory were done on sterile chestnut stems, as described in Liu and Milgroom (1996). Crosses between two isolates were done by placing a mycelial plug of each isolate at opposite ends of the chestnut stem embedded on agar. In tests for selfing, two mycelial plugs from a single isolate were used to inoculate a stem, and the assay proceeded in a manner otherwise identical to that described for biparental crosses.

Fertilizations were effected in the laboratory after 3 weeks. Conidia were dislodged from the agar surrounding the embedded stem using $3 \mathrm{~mL}$ sterile water. The resulting conidial suspension was then pippetted over the colonized chestnut stem. To minimize the risk of fertilizing an isolate on one crossing plate with conidia from other plates (Whitehouse, 1949), all fertilizations were done using aerosol-resistant pipette tips. Additionally, work area, hands, and pipettors were thoroughly wiped down with $70 \%$ ethanol prior to and in between each fertilization. Because contamination would not be detectable between clonal isolates (i.e. isolates with identical DNA-fingerprint genotypes), crosses involving these isolates were not set up or fertilized within a single block of time. Instead, clonal isolates were handled during separate work shifts.

\section{Test for bipolar mating system}

To conclusively demonstrate a bipolar self-incompatibility system, we crossed, in all pairwise combinations (including self-pairings), 20 single-conidial isolates: eight field isolates from China, eight from North America, and four laboratory isolates (strains $\mathrm{Cr} 1 \mathrm{a}, \mathrm{Cr} 1 \mathrm{~b}, \mathrm{Cr} 2 \mathrm{a}$, $\mathrm{Cr} 2 \mathrm{~b}$ ) that were tentatively identified as mating-type tester strains (discussed below) (Table 1). Each pairing was conducted twice.

PCR primers specific to the two idiomorphs of the C. parasitica MAT locus (Marra \& Milgroom, 1999) were used in PCR amplifications to determine if mating-type phenotypes (as determined above) correlated with presence and absence of the two MAT idiomorphs. DNAs extracted from the 20 isolates used in this experiment were used as template in PCR amplifications. Primers and amplification conditions were identical to those described in Marra \& Milgroom (1999).
Table 1 Sources of isolates used in test of bipolar mating system

\begin{tabular}{ll}
\hline Isolate & \multicolumn{1}{c}{ Source } \\
\hline 09135 & Anhui, China \\
09193 & Jiangsu, China \\
09325 & Guandong, China \\
09362 & China \\
09398 & Fujian, China \\
09460 & Jiangsu, China \\
09510 & Anhui, China \\
09555 & Shandong, China \\
BF 33 & Danby, NY, USA \\
GA1 & Georgia, USA \\
KY1 & Kentucky, USA \\
MI33 & Michigan, USA \\
NH1 & New Hampshire, USA \\
NJ124 & New Jersey, USA \\
ON20 & Ontario, Canada \\
WV49 & West Virginia, USA \\
Cr1a & $M A T$-1-tester strain, laboratory* \\
Cr1b & $M A T-1$-tester strain, laboratory \\
Cr2a & $M A T-2$-tester strain, laboratory \\
Cr2b & $M A T$-2-tester strain, laboratory \\
\hline
\end{tabular}

* Development of tester strains is described in the text and Fig. 1.

\section{Mating-type tester strains}

For distinguishing outcrossing from selfing in the laboratory, we developed mating-type tester strains with a mutation for pigment production, which results in cream-coloured (CRE1-) rather than wild-type orange $(C R E 1+)$ mycelium. We used isolate EP389 (ATCC 38980, CRE1-, MAT-2), to cross this marker into strains that would mate well in the laboratory (see Fig. 1). We redefined the mating types in C. parasitica based on the homology of the MAT idiomorphs to those of other ascomycetes (Arie et al., 1997). For example, the mating type of EP155 (ATCC 38755, CRE1+), which was previously reported to be mating type 'A' (Gao \& Nuss, 1996), is now MAT1-2; similarly, mating type 'a' has been renamed MAT1-1. However, because only one MAT locus has been identified in the Ascomycota, the mating types are less formally named $M A T-1$ and MAT-2. The new nomenclature will also supersede the renaming of mating types ' $A$ ' and ' $\mathrm{a}$ ' to MAT1-1 and MAT1-2, respectively, as proposed by Anagnostakis (1988) before MAT homologies were established.

Three generations of crosses were performed, starting with EP389, and continuing with CRE1- progeny, EP155 and wild-type isolates (Fig. 1). From each generation, CRE1- progeny were selected for mating type (determined by both mating assays and 
Fig. 1 Development of cream-coloured (CRE1-) mating-type tester strains. Maternal parent of each cross is underscored. Progeny from crosses are indicated by arrows. See text for sources of isolates.

$M A T$-specific PCR amplifications; see above), vigour (measured as growth on water agar), abundance and viability of conidia (measured as germination on water agar), and male and female fertility in crosses with wildtype isolates. For each cross shown in Fig. 1, three CRE1- progeny of each mating-type were chosen (based on the above criteria, which were judged relatively, not quantitatively). All six progeny were then crossed with wild-type (pigmented) tester strains and other North American isolates to select for CRE1- isolates that produced perithecia most quickly and abundantly. As shown in Fig. 1, CRE1- isolates used for mating-type tests are MAT-1 strains rmx6-1-2, Cr1a and Cr1b, and MAT-2 strains rmx6-2-4, Cr2a and $\mathrm{Cr} 2 \mathrm{~b}$.

\section{Distinguishing self-fertilization from outcrossing}

For most of our assays, the unit of study was the progeny array from a single perithecium, which represents a single mating event (Milgroom et al., 1993). The progeny array within a perithecium is determined to be the result of selfing when there is no segregation of genetic markers (Milgroom et al., 1993). Three sets of genetic markers were used. The first set was relevant only to laboratory crosses between $C R E 1-$ tester strains and $C R E 1+$ (wild-type) isolates. CRE1-/CRE1+ segregation within a progeny array constituted evidence of outcrossing. When progeny from laboratory crosses with a tester strain did not segregate for colour, then the assay for distinguishing selfing and outcrossing was the same as for perithecia from the field, which relied on two sets of markers: vegetative incompatibility loci (Cortesi \& Milgroom, 1998) and DNA fingerprint loci (Milgroom et al., 1992a; Milgroom et al., 1993).

\section{Self-fertilization in the laboratory}

We analysed perithecia from two classes of crossing plates to identify self-fertilization. The first class consisted of crossing plates that were inoculated with only a single isolate. One-hundred and thirty isolates were tested for selfing in this way; 88 were from populations in North America, China, and Europe and 42 were ascospore progeny from laboratory crosses. A minimum of three such crossing plates were set up for each isolate. The second class of crossing plates that were inspected for selfed perithecia was between isolates that were of the same mating-type (as determined by crosses with tester strains), but which had different vic and fingerprint genotypes. These crosses were set up for a variety of reasons, but mainly to determine the mating types (prior to the availability of PCR primers) of various isolates. Presence of perithecia was interpreted as evidence that at least one of the two isolates either had self-fertilized or had been fertilized spuriously by a contaminating conidium from a sexually compatible isolate. Self-fertilization was distinguished from outcrossing by assaying for lack of segregation of genetic markers.

\section{Self-fertilization in the field}

To demonstrate selfing in the field, three isolates, differing in vic and fingerprint genotypes, were inoculated in each of two sites approximately $120 \mathrm{~km}$ apart in the Monongahela National Forest, in the Appalachian Mountains of West Virginia: (1) WV49 was isolated from Clover Run, West Virginia, and was determined to have selfed on that site (Marra, 1998); (2) V7 and (3) V17, from Mt. Lake, Virginia, are isolates from which outcrossed perithecia were previously isolated (Milgroom et al., 1993). All three isolates differed in vic and fingerprint genotypes. None of these three isolates self-fertilized in the laboratory. Both inoculation sites had very low incidence of chestnut blight, so that the resident population of $C$. parasitica was at a low, barely detectable, density. Each isolate was inoculated into five chestnut trees in each site in May 1994. Cankers resulting from these inoculations were sampled for perithecia in October 1995, and November 1996, and progeny arrays were assayed for segregation at vic and DNA-fingerprint loci, as described above. 


\section{Induction of selfing with conidial filtrates}

Evidence for mating-specific pheromone production in C. parasitica (Zhang et al., 1998), as well as in other ascomycete systems (reviewed in Kronstad \& Staben, 1997), prompted us to attempt inducing self-fertilization in $C$. parasitica by using culture filtrates of germinating conidia (which function as male gametes) from isolates of the opposite mating type. Vigfusson \& Cano (1974) were able to induce selfing in Neurospora crassa using sterile filtrates from mating cultures. Although our mating assay would not allow us to obtain filtrates of actively mating cultures, we were able to obtain filtrates of germinating conidia.

Conidia for these experiments came from the $M A T-1$ tester strains rmx6-1-2 and $\mathrm{Cr} 1 \mathrm{~b}$, and from the MAT-2 tester strains rmx6-2-4 and $\mathrm{Cr} 2 \mathrm{~b}$. Cultures grown on PDA were induced to conidiate under artificial light in approximately 10 days. Conidia were harvested by placing $3 \mathrm{~mL}$ sterile distilled water on cultures, loosening conidia with a sterilized rubber spatula, then transferring the conidial suspension to a sterile $1.5-\mathrm{mL}$ tube, after which they were standardized to concentrations of $10^{5}$ conidia $\mathrm{mL}^{-1}$. Conidia were allowed to germinate at room temperature, with moderate agitation, in distilled water that had been autoclaved along with 3-4 segments of chestnut stems (approx. $1 \times 5 \mathrm{~cm}$ ) per litre. Each batch of conidia was allowed to germinate for 24,48 , or $72 \mathrm{~h}$, constituting 12 treatments (four conidial strains $\times$ three germination times). Germination was confirmed by visualizing spore suspensions under a compound microscope $(400 \times)$. To prepare filtrates, the suspensions of germinating conidia were filtered through disposable $0.4 \mu \mathrm{m}$ SFCA filters (Nalge Nunc International, Rochester, NY). An aliquot of each filtrate was placed on PDA and inspected over at least five days to ascertain that the filtrates contained no viable conidia.

We attempted to artificially induce selfing in five isolates that had apparently selfed in nature (unpublished results): BF12-m1, DU8-m, V38,c5, WV43,c1 and WV49,c1. Each isolate was placed alone on a chestnut stem in mating medium and allowed to colonize it, as described above. Each of the five isolates was then 'mock fertilized' with 2-mL aliquots of each of the 12 sterile culture filtrates of germinating conidia, described above. We also fertilized with the following two controls: (1) $2-\mathrm{mL}$ aliquots of each of the 12 treatments, prior to filtration, to confirm that the germinating conidia were themselves fertile; and (2) 2-mL aliquots of sterile distilled water, to control for selfing that might occur in the absence of culture filtrates. Because laboratory matings in $C$. parasitica require chestnut stems, the sterile distilled water used in (2) was autoclaved with chestnut stems as described above.
Beginning two weeks after fertilizations and for 8 weeks thereafter, plates were checked weekly for perithecia. Perithecia were sampled, ascospores isolated, and assayed for selfing as described above.

\section{Results}

\section{Bipolar self-incompatibility system}

Pairwise matings among 20 isolates resulted in all isolates belonging to either one incompatibility group or the other (Fig. 2). Although fertility between North America and China, as well as fertility within China, was lower than fertility within North America (results not shown), nonetheless the 16 field isolates could be divided into two groups based on sexual compatibility: isolates in one group are sexually compatible with isolates in the other group, whereas isolates within a group are sexually incompatible with each other. Nearly all crosses that were scored as being fertile produced abundant $(>50)$ perithecia. However, sparse perithecia (fewer than five per replicate crossing plate) were found on several crosses that were between isolates of the same mating-type. In none of these cases were perithecia found in both replicate pairings. Additionally, only one of the isolates in any of these pairings produced perithecia.

Each $M A T$-specific PCR primer pair amplified DNA fragments of the expected sizes from isolates of only one compatibility group, and not the other (Fig. 3). MAT-1specific primers M1-GS1 and M1-GS2-rev (Marra \& Milgroom, 1999) amplified the expected 2.2-kb product only from isolates that were phenotypically MAT-1. Similarly, $M A T$-2-specific primers M2-GS2 and InvA5n (Marra \& Milgroom, 1999) amplified the expected $1.7-\mathrm{kb}$ product only from isolates that were phenotypically $M A T-2$, with the exception of isolate GA1, which was lost and therefore not tested.

\section{Self-fertilization in the laboratory}

Of the 427 crossing plates that were inoculated with single isolates, nine contained a total of 44 perithecia; of these, 22 were shown to be the result of self-fertilization. All 22 selfed perithecia were the result of selfing by isolates derived clonally (i.e. asexual conidia or ascospores resulting from selfing) from one genotype, WV43 (Fig. 4): WV43-1-3 (an ascospore progeny from selfing by WV43 in nature); A2-17-5 (an ascospore progeny from selfing in the laboratory by WV43-1-3); and A2-175,c5 (a single conidial isolate of A2-17-5). The remaining 22 perithecia were outcrossed - i.e. progeny from each of these perithecia segregated at vic and/or DNAfingerprint loci - presumably due to fertilization by contaminating conidia. 


\begin{tabular}{|c|c|c|c|c|c|c|c|c|c|c|c|c|c|c|c|c|c|c|c|c|c|}
\hline & \multicolumn{9}{|c|}{ MAT-1 } & \multicolumn{5}{|c|}{ Female Parents } & \multicolumn{6}{|c|}{ MAT-2 } \\
\hline & & 09135 & 09193 & 09362 & 09398 & 09460 & BF33 & $\mathrm{KY} 1$ & MI33 & Cria & Cr1b & 09325 & 09510 & 09555 & GA1 & $\mathrm{NH1}$ & \begin{tabular}{|l|} 
NJ124 \\
\end{tabular} & ON20 & WV49 & \begin{tabular}{|c|}
$\mathrm{Cr} 2 \mathrm{a}$ \\
\end{tabular} & $\mathrm{Cr} 2 \mathrm{~b}$ \\
\hline \multirow{8}{*}{ 市 } & $\lcm{09135}$ & & & & & & & & & & & & + & + & ++ & + & ++ & + & ++ & ++ & ++ \\
\hline & 09193 & & & & & & & & & & & + & + & $\{+\}$ & ++ & ++ & ++ & ++ & ++ & ++ & \\
\hline & 09362 & & & & & & & & & & & ++ & ++ & ++ & ++ & ++ & ++ & ++ & ++ & & ++ \\
\hline & 09398 & & & & & & & & & $\{+\}$ & & + & ++ & $\{+\}$ & ++ & ++ & ++ & ++ & ++ & & ++ \\
\hline & 09460 & & & & & & & & & & & & ++ & & ++ & ++ & ++ & ++ & ++ & ++ & \\
\hline & $\overline{B F 33}$ & & & & & & & & $\{+\}$ & & & & ++ & + & + & ++ & ++ & ++ & ++ & & ++ \\
\hline & KY1 & & & & & & & & & & & ++ & ++ & ++ & ++ & ++ & ++ & ++ & ++ & ++ & ++ \\
\hline & MI 33 & & & & & & $\{+\}$ & & & & & & ++ & + & ++ & ++ & ++ & ++ & ++ & ++ & ++ \\
\hline \multirow{12}{*}{ 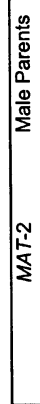 } & Cr1a & & & $\{+\}$ & & & & & & & & ++ & ++ & ++ & ++ & ++ & ++ & ++ & ++ & ++ & ++ \\
\hline & Crib & & & & & & & & & & & & ++ & ++ & ++ & ++ & ++ & & ++ & ++ & ++ \\
\hline & 09325 & + & $\begin{array}{l}+ \\
+\end{array}$ & ++ & 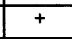 & ++ & ++ & ++ & ++ & & & & & & & & & & & & \\
\hline & 09510 & & & ++ & + & + & ++ & ++ & ++ & ++ & & & & & & & & & & & \\
\hline & 09555 & & $\{+\}$ & ++ & $\{+\}$ & & ++ & ++ & ++ & ++ & ++ & & & & & & & & & & \\
\hline & GA1 & $\begin{array}{c}+ \\
\end{array}$ & + & + & + & ++ & + & ++ & + & ++ & ++ & & & & & & & & & & \\
\hline & $\mathrm{NH1}$ & + & & + & & + & ++ & ++ & + & ++ & ++ & & $\{+\}$ & & & & & & $\{+\}$ & & \\
\hline & NJ124 & $\{+\}$ & + & ++ & + & ++ & ++ & ++ & ++ & ++ & ++ & & & & & & & & & & \\
\hline & ON20 & & + & ++ & + & - & ++ & ++ & ++ & ++ & & & & & & & & & & & \\
\hline & WV49 & & & ++ & ++ & ++ & ++ & ++ & ++ & ++ & ++ & & & & & & & & & & \\
\hline & Cr2a & & ++ & ++ & & ++ & & ++ & ++ & ++ & ++ & & & & & & & & & & \\
\hline & Cr2b & + & ++ & ++ & ++ & ++ & ++ & ++ & ++ & ++ & ++ & & & & & & & & & & \\
\hline
\end{tabular}

Fig. 2 Test for bipolar mating system. Sources for parental isolates are shown in Table 1. Cr1a and Cr1b are MAT-1-tester strains; $\mathrm{Cr} 2 \mathrm{a}$ and $\mathrm{Cr} 2 \mathrm{~b}$ are $M A T$-2-tester strains (see text and Fig. 1). Each pairing was done in two replicates. ' + ' indicates that perithecia were observed in one replicate pairing; ' ++ ' indicates that perithecia were observed in both replicates. Scores in parentheses indicate that there were fewer than 5 perithecia per isolate per plate; almost all other ' ++ ' crosses produced 50 or more perithecia. Empty cells indicate that no perithecia were observed.

We also looked for selfed perithecia from crosses attempted between isolates of the same mating-type. We sampled a total of 202 perithecia, isolated from 35 of these crossing plates (including all seven perithecia from six crossing plates indicated in Fig. 2) and assayed each for marker segregation. One-hundred and sixty-five of these 202 perithecia were the results of outcrossing. However, we identified 37 perithecia whose progeny arrays did not segregate for any markers. These perithecia represent self-fertilization by only 10 isolates, five of which were members of the WV43 clonal lineage, described above (and shown in Fig. 4). The remaining five isolates that self-fertilized on these crossing plates are: ascospore isolates BF15-4-5 and DU85-4-4; singleconidial isolates BF15-4-6,c2 and rmx6-1-2,c1 (both derived from ascospore isolates); and maternal isolate DU73-4-m.

Self-fertilization is not restricted to a single mating type. Both mating types (MAT-1 and MAT-2) were represented by these six self-fertile genotypes. Based on mating assays and $M A T$-specific PCR amplifications (results not shown), DU85-4-4, rmx6-1-2,c1 and the WV43 clonal lineage are $M A T-1$, and BF15-4-5, BF154-6,c2 and DU73-4-m are MAT-2.

\section{Self-fertilization in nature}

Self-fertilized perithecia were obtained from all three of the inoculated genotypes, in both sites (Table 2).
Self-fertilization occurred in both one-year-old and two-year-old cankers. Several cankers from all three inoculated genotypes contained both selfed and outcrossed perithecia (Table 2).

\section{Induction of selfing}

We were not able to induce selfing in any of the five isolates with sterile filtrates of germinating conidia taken from isolates of the opposite mating-type. Germination was confirmed to have occurred in a majority of conidia in suspensions after 24-h, 48-h, and 72-h incubation times. Each of the five isolates was successfully fertilized by germinating conidia (i.e. prefiltration) of the opposite mating-type, for all three incubation times. When filtrates were plated onto PDA and allowed to incubate, no growth was observed after five days, verifying that filtrates contained no viable conidia.

\section{Discussion}

This report demonstrates that self-incompatibility and self-fertilization are two important - and seemingly contradictory - aspects of $C$. parasitica's reproductive biology. Our conclusion that $C$. parasitica has a bipolar self-incompatibility system, resembling the mating systems of other self-incompatible ascomycetes, is based on the results of all pairwise matings among 20 isolates, from which we observed two alleles segregating at a 
(a)

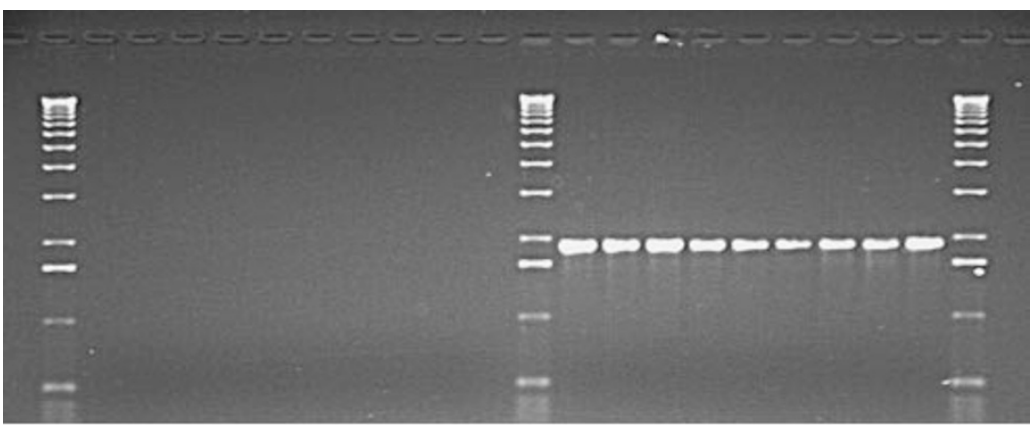

$\begin{array}{llllllllllllllllllllll}1 & 2 & 3 & 4 & 5 & 6 & 7 & 8 & 9 & 10 & 11 & 12 & 13 & 14 & 15 & 16 & 17 & 18 & 19 & 20 & 21 & 22\end{array}$

(b)

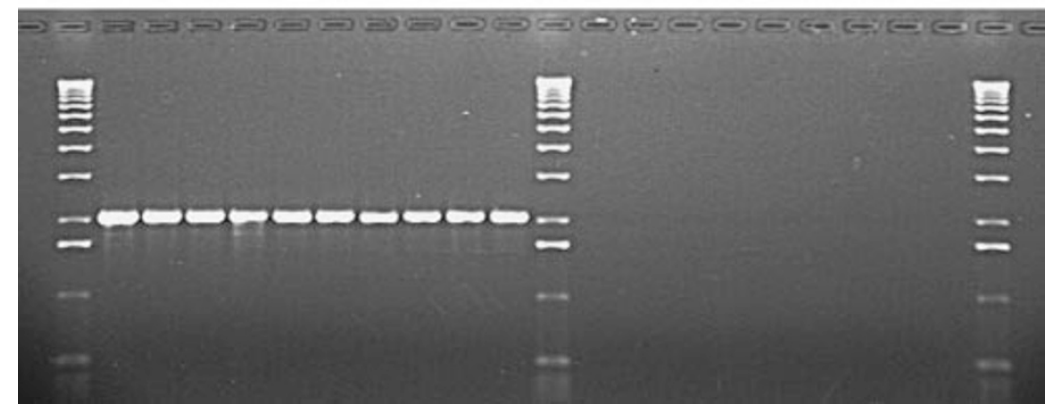

Fig. 3 PCR amplifications from $M A T-1$ (lanes 2-11) and $M A T$-2 (lanes 13-21) isolates (Table 1 and Fig. 2) using (a) $M A T$-2-specific primers, and (b) $M A T-1$ specific primers (Marra \& Milgroom, 1999). Lanes 1,12 and 22 contain $1-\mathrm{kb}$ ladder. Other lanes and isolates are as follows: lane 2, Cr1a; lane 3, Cr1b; lane 4, 09135; lane 5, 09193; lane 6, 09362; lane 7, 09398; lane 8, 09460; lane 9, BF33; lane 10, KY1; lane 11, MI33; lane 13, Cr2a; lane 14, Cr2b; lane 15, 09325; lane 16, 09510; lane 17, 09555; lane 18, $\mathrm{NH} 1$; lane 19, NJ124; lane 20, ON20; lane 21, WV49. Note that $M A T-1$ primers amplify the expected $2.2-\mathrm{kb}$ product only from phenotypically $M A T-1$ isolates, and $M A T-2$ primers amplify the expected $1.7-\mathrm{kb}$ product only from phenotypically $M A T-2$ isolates.

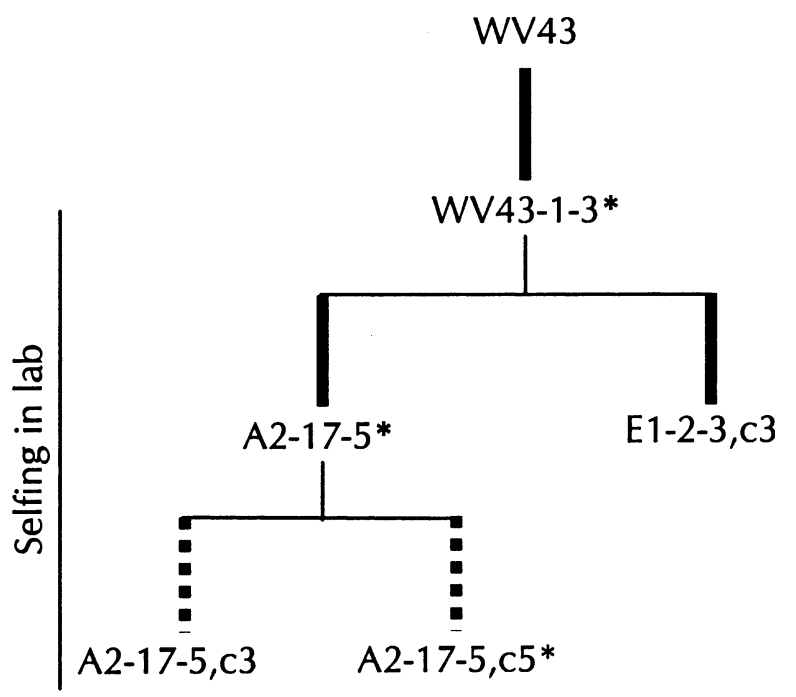

Fig. 4 Self-fertilization in the clonal lineage derived from field isolate WV43 (which was determined to have selfed in nature, unpublished). Solid bars indicate self-fertilization (resulting in ascospores). Dashed bars indicate asexual reproduction (resulting in conidia). Isolates indicated by '*' are those that selfed when placed alone on mating medium, and in the presence of isolates of the same mating-type; isolates E1-2-3,c3 and A2-17-5,c3 selfed in the presence of other isolates of the same mating type but not when placed alone on mating medium.

single locus $(M A T)$. Furthermore, PCR markers for mating-type idiomorphs correlated perfectly with assignments to mating-types based on mating pheno- types in the crossing study. From these studies, we have developed four mating-type tester strains, two of each mating-type ( $M A T-1$ and $M A T-2)$, employing a marker based on a single-locus pigment mutation (CRE1-). Although self-incompatibility has been assumed in earlier discussions of $C$. parasitica's mating system (Anagnostakis, 1984, 1988; Liu et al., 1996), this is the first report to conclusively demonstrate the selfincompatibility system in $C$. parasitica.

The second important aspect of $C$. parasitica's reproductive biology demonstrated here is the potential for self-fertilization in both the laboratory and field. Selfing is a rare and unpredictable phenomenon in the laboratory. It is interesting to note that of the 10 isolates that self-fertilized in our laboratory, five are ramets of a clonal lineage derived from a canker isolate, WV43, that selfed in nature. Though all five of these ramets selfed in the laboratory, they did so extremely rarely, and otherwise behaved predictably as $M A T-1$ isolates. The ability to self-fertilize, then, appears not to be associated with an imperfectly functioning self-incompatibility system. However, that some isolates self-fertilize in the laboratory, while others do not, suggests that there are genetic differences in the proclivity to self. Self-fertilization also appears to be associated with environmental factors, as is suggested by our finding that three isolates, none of which was self-fertile in the laboratory, selfed readily in nature.

In previous reports, selfing in natural populations was inferred from the absence of marker segregation among sibling progeny (Milgroom et al., 1993). The markers 
Table 2 Self-fertilization in nature by inoculated genotypes of $C$. parasitica

\begin{tabular}{|c|c|c|c|c|c|}
\hline \multirow{3}{*}{$\begin{array}{l}\text { Inoculated } \\
\text { genotype } \dagger \\
\text { (mating type) }\end{array}$} & \multirow[b]{3}{*}{ Canker } & \multicolumn{4}{|c|}{ Perithecia* } \\
\hline & & \multicolumn{2}{|c|}{1995} & \multicolumn{2}{|c|}{1996} \\
\hline & & Selfed & Outcrossed & Selfed & Outcrossed \\
\hline \multirow{3}{*}{$\begin{array}{l}\text { WV49 } \\
\qquad(M A T-2)\end{array}$} & 1 & $-\S$ & - & 1 & 1 \\
\hline & 2 & - & - & 2 & 1 \\
\hline & 3 & - & - & 1 & 4 \\
\hline \multirow{3}{*}{$\begin{array}{l}\mathrm{V} 7, \mathrm{c} 2 \\
\quad(M A T-1)\end{array}$} & 1 & 1 & 1 & 0 & 1 \\
\hline & 2 & 1 & 2 & 1 & 0 \\
\hline & 3 & 1 & 0 & 0 & 1 \\
\hline \multirow{3}{*}{$\begin{array}{l}\mathrm{V} 17, \mathrm{c} 5 \\
\quad(M A T-2)\end{array}$} & 1 & 0 & 1 & 1 & 0 \\
\hline & 2 & 1 & 1 & 0 & 1 \\
\hline & 3 & 1 & 0 & 0 & 1 \\
\hline
\end{tabular}

* A minimum of 15 progeny per perithecium was assayed for segregation at vic loci, and a minimum of 8 progeny per perithecium was assayed for segregation at DNA fingerprint loci.

$\dagger$ Inoculations were done in spring of 1994, one inoculation per tree; therefore, each canker is considered an independent replicate. Perithecia were sampled in October 1995 and November 1996.

$\$$ Mating types determined by crosses with mating-type tester strains.

$\S \mathrm{WV}-49, \mathrm{ml}$ inoculations were not sampled in 1995. used had been shown to be highly polymorphic in the populations studied. However, subsequent reports of biparental inbreeding (Marra \& Milgroom, 1994) and spatial genetic structure (Milgroom \& Lipari, 1995b) suggest that progeny determined to be the products of self-fertilization may actually be the products of mating between closely inbred parents. In this report, we conclude that self-fertilization, rather than biparental inbreeding, occurred based on the following: (1) the inoculated isolates each had different vic and fingerprint genotypes; (2) isolates were inoculated into sites that at the time of inoculation showed little or no evidence of chestnut blight; (3) vic and DNA fingerprint genotypes are highly polymorphic in north-eastern U.S. populations of C. parasitica (Milgroom et al., 1992a, 1993; Liu et al., 1996; Milgroom \& Cortesi, 1999), such that a resident genotype is extremely unlikely to be identical to an inoculated genotype from a different population; and (4) selfing was observed in all three genotypes, further reducing the probability of biparental inbreeding with resident genotypes.

Several pairings of isolates (Fig. 2) produced sparse $(<5)$ perithecia. In some cases, these conformed to expectation; i.e. they were between isolates of opposite mating-type. The most obvious explanation for the poor fertility in these crosses is that the mating assay that we use is vagarious; isolates that cross abundantly on one replicate plate may cross poorly or not at all on another. We have made several attempts to standardize a more homogeneous mating assay, but the placement of scarified autoclaved dormant chestnut stems in water agar remains the most productive and consistent method for this fungus (unpublished observations).

We also observed perithecia in 35 pairings that were between isolates of the same mating-type (where mating types were determined by crosses with mating-type tester strains). In all of these cases, the perithecia were sparse (fewer than five per plate), and only one of the two replicate pairings produced any perithecia at all. Surprisingly, most of these perithecia were the result of outcrossing, and were assumed to be the result of contamination by conidia from other sexually compatible isolates.

We were unable to induce selfing in $C$. parasitica by using sterile filtrates of germinating conidia of the opposite mating-type. In $N$. crassa, sex pheromones, which are under the control of the MAT locus, mediate the attraction between conidia and trichogynes (female receptive hyphae) of opposite mating-type (Bistis, 1981; Bistis, 1983), a necessary precedent to plasmogamy. Even earlier work on $N$. crassa showed that a single haploid isolate was capable of self-fertilizing in the presence of sterile diffusible extracts from cultures of the opposite mating type (Vigfusson \& Cano, 1974). One interpretation of this result is that both mating types are necessary for completion of the sexual cycle. However, several self-fertile Neurospora spp. have been shown to have only one or the other idiomorph (Glass et al., 1988; Glass et al., 1990).

It is perhaps paradoxical, given the evidence for a genetically determined self-incompatibility system, that $C$. parasitica self-fertilizes so frequently in nature. The 
mechanism by which self-incompatibility is bypassed in order to permit self-fertilization is currently under investigation. $C$. parasitica presents several additional challenges for understanding the biology and evolution of mating systems. For example, our current research explores the mating system of $C$. parasitica from three additional perspectives: (1) the effect of the mixed-mating system, in terms of selfing rates and inbreeding estimates, on population genetic structure; (2) the relative contributions of environment and genetics in determining an individual's propensity to self-fertilize or outcross in natural populations; and (3) the role of inbreeding depression in maintaining a mixed mating system in $C$. parasitica. Further investigation in this area is warranted, as a genetically variable determinant of self-fertilization would have profound consequences on the response to selection and therefore could contribute significantly to theories on the evolution of self-fertilization and mixed mating systems.

\section{Acknowledgements}

The authors thank Y.-C. Liu and I.C. Agudelo for general assistance in the laboratory. We thank W.L. MacDonald and M.L. Double for help in identifying inoculation sites in West Virginia. We also thank J. Kohn for reviewing an earlier draft of this manuscript. Financial support came, in part, from United States Department of Agriculture National Research Initiative Competitive Grant 93-37303-9035, National Science Foundation Grant DEB-9707675, and McIntire-Stennis Project NYC-153553.

\section{References}

AINSWORTH, A. M. 1987. Occurrence and interactions of outcrossing and non-outcrossing populations in Stereum, Phanerochaete, and Coniophora. In: Rayner, A. D. M., Brasier, C. M. and Moore, D. (eds) Evolutionary Biology of the Fungi. Cambridge University Press, Cambridge.

ALEXopoulos, C. J., MIMS, C. W. AND BLACKWELL, M. 1996. Introductory Mycology, 4th edn. John Wiley \& Sons, New York.

ANAGNOSTAKIS, S. L. 1984. The mycelial biology of Endothia parasitica II. Vegetative incompatibility. In: Jennings, D. H. and Rayner, A. D. M. (eds) The Ecology and Physiology of the Fungal Mycelium, pp. 499-507. Cambridge University Press, Cambridge.

AnAgnostakis, S. L. 1987. Chestnut blight: the classical problem of an introduced pathogen. Mycologia, 79, 23-37.

ANAGNOSTAKIS, S. L. 1988. Cryphonectria parasitica, cause of chestnut blight. In: Sidhu, G. S., Ingram, D. S. and Williams, P. H. (eds) Genetics of Plant Pathogenic Fungi, vol. 6, pp. 123-136. Academic Press, New York.
ARIE, T., CHRISTIANSEN, S. K., YODER, O. C. AND TURGEON, B. G. 1997. Efficient cloning of ascomycete mating-type genes by PCR amplification of the conserved MAT HMG box. Fungal Gen. Biol., 21, 118-130.

BARRETT, S.C. H. 1988. The evolution, maintenance, and loss of self-incompatibility systems. In: Doust, J. L. and Doust, L. L. (eds) Plant Reproductive Ecology: Patterns and Strategies, pp. 98-124. Oxford University Press, New York.

BARRETT, S. C. H. AND ECKERT, C. G. 1990. Variation and evolution of mating systems in seed plants. In: Kawano, S. (ed.) Biological Approaches and Evolutionary Trends in Plants, pp. 229-254. Academic Press, London.

BISTIS, G. N. 1981. Chemotropic interactions between trichogynes and conidia of opposite mating type in Neurospora crassa. Mycologia, 73, 959-975.

BISTIS, G. N. 1983. Evidence for diffusible, mating-type-specific trichogyne attractants in Neurospora crassa. Exp. Mycol., 7 , 292-295.

COPPIN, E., DEBUChY, R., ARNAISE, S. AND PICARD, M. 1997. Mating types and sexual development in filamentous ascomycetes. Microbiol. Mol. Biol. Rev., 61, 411-428.

CORTESI, P. AND MILGROOM, M. G. 1998. Genetics of vegetative incompatibility in Cryphonectria parasitica. Appl. Environ. Microbiol., 64, 2988-2994.

CORTESI, P., MILGROOM, M. G. AND BISIACH, M. 1996. Distribution and diversity of vegetative compatibility types in subpopulations of Cryphonectria parasitica in Italy. Mycol. Res., 100, 1087-1093.

ENNOS, R. A. AND SwALES, K. W. 1987. Estimation of the mating system in a fungal pathogen Crumenulopsis sororia (Karst.) Groves using isozyme markers. Heredity, 59, 423-430.

GAO, S. AND NUSS, D. L. 1996. Distinct roles for two G protein subunits in fungal virulence, morphology, and reproduction revealed by targeted gene disruption. Proc. Natl. Acad. Sci. U.S.A., 93, 14122-14127.

GLASS, N. L., VOllmer, S. J., STABEN, C., GROTELUESCHEN, J. ET AL. 1988. DNAs of the two mating-type alleles of Neurospora crassa are highly dissimilar. Science, 241, 570-573.

GLASS, N. L., METZENBERG, R. AND RAJU, N. B. 1990. Homothallic Sordariaceae from nature: the absence of strains containing only the a mating type sequence. Exp. Mycol., 14, 274-289.

GRIFFIN, D. H., QUINN, K. E., GILBERT, G. S., WANG, C. J. K. ET $A L$. 1992. The role of ascospores and conidia as propagules in the disease cycle of Hypoxylon mammatum. Phytopathology, 82, 114-119.

HEDRICK, P. W. 1990. Mating systems and evolutionary genetics. In: Wohrmann, K. and Jain, S. (eds) Population Biology, Ecological and Evolutionary Viewpoints, pp. 83-114. Springer, Berlin.

JARNE, P. AND CHARLESWORTH, D. 1993. The evolution of the selfing rate in functionally hermaphrodite plants and animals. Ann. Rev. Ecol. Syst., 24, 441-466.

KOHLI, Y. AND KOHN, L. M. 1992. In vitro and in vivo outbreeding studies on Sclerotinia sclerotiorum. Newsl. Mycol. Soc. Am., 43, 39 (Abstract).

KRONSTAD, J. W. AND STABEN, C. 1997. Mating type in filamentous fungi. Ann. Rev. Genet., 31, 245-276. 
KUES, U. AND CASSELTON, L. A. 1992. Fungal mating type genes regulators of sexual development. Mycol. Res., 12, 993-1006.

LEUCHTMANN, A. AND CLAY, K. 1989. Isozyme variation in the fungus Atkinsonella hypoxylon within and among populations of its host grasses. Can. J. Bot., 67, 2600-2607.

LIU, Y.-C. AND MILGRoOM, M. G. 1996. Correlation between hypovirus transmission and the number of vegetative incompatibility (vic) genes different among isolates from a natural population of Cryphonectria parasitica. Phytopatho$\log y, \mathbf{8 6}, 79-86$.

MARRA, R. E. 1998. Selfing in the Context of Self-Incompatibility: the Mixed Mating System of the Fungus Cryphonectria parasitica. PhD Thesis, Cornell University.

MARRA, R. E. AND MILGROOM, M. G. 1994. Selfing and outcrossing in the chestnut blight fungus (Cryphonectria parasitica) in natural populations. Phytopathology, 84, 1974.

MARRA, R. E. AND MILGROOM, M. G. 1999. PCR amplification of the mating-type idiomorphs in Cryphonectria parasitica. Mol. Ecol., 8, 1947-50.

MILGROOM, M. G. AND CORTESI, P. 1999. Analysis of population structure of the chestnut blight fungus based on vegetative incompatibility genotypes. Proc. Natl. Acad. Sci. U.S.A., 96, 10518-10523.

MILGROOM, M. G. AND LIPARI, S. E. 1995a. Population differentiation in the chestnut blight fungus, Cryphonectria parasitica, in eastern North America. Phytopathology, 85, 155-160.

MILGROOM, M. G. AND LIPARI, S. E. 1995b. Spatial analysis of nuclear and mitochondrial RFLP genotypes in populations of the chestnut blight fungus, Cryphonectria parasitica. Mol. Ecol., 4, 633-642.

MILGROOM, M. G., LIPARI, S. E. AND POWELL, w. A. 1992a. DNA fingerprinting and analysis of population structure in the chestnut blight fungus, Cryphonectria parasitica. Genetics, 131, 297-306.

MILGROOM, M. G., LIPARI, S. E. AND WANG, K. 1992b. Comparison of genetic diversity in the chestnut blight fungus, Cryphonectria (Endothia) parasitica from China and the US. Mycol. Res., 96, 1114-1120.
MILGROOM, M. G., LIPARI, S. E., ENNOS, R. A. AND LIU, Y.-C. 1993. Estimation of the outcrossing rate in the chestnut blight fungus, Cryphonectria parasitica. Heredity, 70, 385-392.

MILGROOM, M. G., WANG, K., ZHOU, Y., LIPARI, S. E. ET AL. 1996. Intercontinental population structure of the chestnut blight fungus, Cryphonectria parasitica. Mycologia, 88, 179-190.

NELSON, M. A. 1996. Mating systems in ascomycetes: a romp in the sac. Trends Genet., 12, 69-74.

PUhAlla, J. E. AND ANAGNOSTAKis, s. L. 1971. Genetics and nutritional requirements of Endothia parasitica. Phytopathology, 61, 169-173.

RAPER, J. R. 1966. Life cycles, basic patterns of sexuality, and sexual mechanisms. In: Ainsworth, G. C. and Sussmann, A. S. (eds) The Fungi, vol. II. The Fungal Organism (an Advanced Treatise), pp. 473-511. Academic Press, New York.

RAYNER, A. D. M. AND TURTON, M. N. 1982. Mycelial interactions and population structure in the genus Stereum: S. rugosum, S. sanguinolentum and S. rameale. Trans. Br. Mycol. Soc., 78, 483-493.

RICHARDS, A. J. 1997. Plant Breeding Systems, 2nd edn. Chapman \& Hall, London.

SHARLAND, P. R. AND RAYNER, A. D. M. 1989a. Mycelial interactions in outcrossing populations of Hypoxylon. Mycol. Res., 93, 187-198.

SHARLAND, P. R. AND RAYNER, A. D. M. 1989b. Mycelial ontogeny and interactions in non-outcrossing populations of Hypoxylon. Mycol. Res., 93, 273-281.

VIGfusson, N. V. AND CANO, R. J. 1974. Artificial induction of the sexual cycle of Neurospora crassa. Nature, 249, 383-385.

WHITEHOUSE, H. L. K. 1949. Heterothallism and sex in the fungi. Biol. Rev., 24, 411-447.

WRIGHT, S. 1946. Isolation by distance under diverse systems of mating. Genetics, 141, 39-59.

ZHANG, L., BAASIRI, R. A. AND VAN ALFEN, N. K. 1998. Viral repression of fungal pheromone precursor gene expression. Mol. Cell. Biol., 18, 953-959. 